\title{
Chlorhexidine-related ventilator-associated events: Toward recognition?
}

\author{
Mieke Deschepper ${ }^{1}$, Dirk Vogelaers ${ }^{2}$ and Stijn Blot RN, MW, MNSc, PhD $^{2,3}$ \\ ${ }^{1}$ Strategic Policy Cell, Ghent University Hospital, Ghent, Belgium, ${ }^{2}$ Department of Internal Medicine, Ghent University, Ghent, Belgium and ${ }^{3}$ Burns, Trauma, and \\ Critical Care Research Centre, The University of Queensland, Center for Clinical Research, Faculty of Medicine, Brisbane, Australia
}

To the Editor-Despite all the efforts in prevention, the concept of ventilator-associated events (VAEs), including but not restricted to ventilator-associated pneumonia, remains an important quality indicator in critically ill patients because these bundled events are associated with increased morbidity and possibly mortality. ${ }^{1-3}$ Ventilator care bundles have been widely implemented to target the optimization of adherence with evidence-based prevention measures. In a recent issue of Infection Control and Hospital Epidemiology, Harris et $\mathrm{al}^{4}$ explored the impact of ventilator bundle compliance on the risk of VAE by means of nested case-control study.

Control subjects were matched for type of ICU and exposure time on the ventilator prior to the VAE. Total bundle compliance was $73 \%$ for cases and was $70 \%$ for controls; compliance to individual components of the bundle ranged from $83 \%$ to $99 \%$. No difference in VAE risk was observed between cases and controls, probably because of the insignificant differences in compliance between the 2 groups. Interestingly, however, higher compliance with chlorhexidine oral care (in the 3 days preceding the event) was associated with a higher risk for VAE in multivariate analysis adjusted for age and sex (odds ratio, 1.45; 95\% confidence interval, 1.10-1.90). Restricting the analysis to infection-related ventilator-associated complications and probable ventilator-associated pneumonia revealed identical results.

As the authors themselves point out, the data must be interpreted cautiously because no adjustment was obtained for severity of illness. Nevertheless, this observation adds to the mounting controversy about chlorhexidine oral care and the suggestion of possible harm. Two meta-analyses of randomized controlled trials have indicated a potential link between chlorhexidine oral care and mortality. ${ }^{5,6}$ A retrospective cohort study evaluating associations between ventilator bundle components and outcomes found chlorhexidine oral care to be associated with increased ventilator mortality. ${ }^{7}$ However, this observation is complicated by the absence of a convincing pathogenic mechanism. Microaspiration of chlorhexidine along folds in the endotracheal cuff wall resulting in VAEs has been proposed as an explanation, albeit without obvious proof., ${ }^{5,8,9}$ Recently, Deschepper et $\mathrm{al}^{10}$ found increased mortality among patients exposed to chlorhexidine oral care in a large hospital-wide cohort, thereby broadening the scope of the issue to all hospitalized patients. ${ }^{10}$ Notably, in this study, no adverse effect of chlorhexidine oral care was observed among ICU patients, either ventilated or nonventilated. Actually,

Author for correspondence: Stijn Blot, Department of Internal Medicine, Ghent University, Campus UZ Gent, Corneel Heymanslaan 10, 9000 Ghent, Belgium. E-mail: stijn.blot@UGent.be

Cite this article: Deschepper M, et al. (2018). Chlorhexidine-related ventilatorassociated events: Toward recognition?. Infection Control \& Hospital Epidemiology 2018, 39, 1144-1145. doi: 10.1017/ice.2018.173 the association of chlorhexidine oral care with mortality was strongest in categories with lower risk of mortality. In this regard, the study by Deschepper et al contradicts previous reports and thereby undermines the hypothesis of microaspiration being the pathogenic mechanism. Of course, the Deschepper study was observational and, as such, had a high risk of bias. ${ }^{11}$ However, in the light of accumulating reports warning of potential harm associated with chlorhexidine oral care, it is time to carefully reconsider this practice. Bouadma and Klompas stressed the relatively low evidence for the use of chlorhexidine in terms of pneumonia reduction. ${ }^{12}$ Indeed, chlorhexidine oral care has been perceived as inexpensive, logical, and completely safe, favoring promotion of the practice. However, the situation turns with increasing indications of associations with potentially deleterious effects. It is correct that warnings about safety of chlorhexidine oral care are derived from meta-analyses and observational cohorts, but how much evidence do we need to omit a potentially harmful practice for which the evidence in favor is also marginal? A significantly reduced risk of pneumonia has only been demonstrated in cardio-surgical patients, and when meta-analyses were restricted to blinded trials, no difference remained. ${ }^{5,12,13}$

Several alternatives are available for chlorhexidine mouthwashes. Thus far, none of these have proven efficacious in reducing pneumonia risk. However, antiseptic mouthwash does have other indications as well, such as the care for patients with stomatitis or gingival-periodontal disease. Also, for these indications, the use of chlorhexidine should be questioned. In any case, oral care either in critically or noncritically ill populations needs to be reassessed and given new attention in research. Solid standard oral care, including 2-3 times daily mechanical cleaning and/or toothbrushing without the use of antiseptics, must be the starting basis for evaluation, against which the benefit of any other additional intervention should be measured.

\section{Acknowledgments}

Financial support. S.B. holds a research mandate of the Special Research Fund at Ghent University. No other financial support was provided relevant to this article.

Conflicts of interest. All authors declare that there are no potential conflicts of interest to disclose.

\section{References}

1. Klompas M, Branson R, Eichenwald EC, et al. Strategies to prevent ventilator-associated pneumonia in acute care hospitals: 2014 update. Infect Control Hosp Epidemiol 2014;35:915-936.

2. Sousa AS, Ferrito C, Paiva JA. Intubation-associated pneumonia: an integrative review. Intensive Crit Care Nurs 2018;44:45-52. 
3. Myny D, Depuydt P, Colardyn F, Blot S. Ventilator-associated pneumonia in a tertiary care ICU: analysis of risk factors for acquisition and mortality. Acta Clin Belg 2005;60:114-121.

4. Harris BD, Thomas GA, Greene MH, Spires SS, Talbot TR. Ventilator bundle compliance and risk of ventilator-associated events. Infect Control Hosp Epidemiol 2018;39:637-643.

5. Klompas M, Speck K, Howell MD, Greene LR, Berenholtz SM. Reappraisal of routine oral care with chlorhexidine gluconate for patients receiving mechanical ventilation: systematic review and meta-analysis. JAMA Intern Med 2014;174:751-761.

6. Price R, MacLennan G, Glen J, SuDDICU Collaboration. Selective digestive or oropharyngeal decontamination and topical oropharyngeal chlorhexidine for prevention of death in general intensive care: systematic review and network meta-analysis. BMJ 2014;348(Mar 31 2):g2197-g2197.

7. Klompas M, Li L, Kleinman K, Szumita PM, Massaro AF. Associations between ventilator bundle components and outcomes. JAMA Intern Med 2016;176:1277-1283.
8. Blot SI, Poelaert J, Kollef M. How to avoid microaspiration? A key element for the prevention of ventilator-associated pneumonia in intubated ICU patients. BMC Infect Dis 2014;14:119.

9. Bouadma L, Karpanen T, Elliott T. Chlorhexidine use in adult patients on ICU. Intensive Care Med 2018;30(Suppl 1):S827-S823.

10. Deschepper M, Waegeman W, Eeckloo K, Vogelaers D, Blot S. Effects of chlorhexidine gluconate oral care on hospital mortality: a hospital-wide, observational cohort study. Intensive Care Med 2018; 37:2709-2710.

11. Ricard JD, Lisboa T. Caution for chlorhexidine gluconate use for oral care: insufficient data. Intensive Care Med 2018;11:845-843.

12. Bouadma L, Klompas M. Oral care with chlorhexidine: beware! Intensive Care Med 2018;35(Suppl 2):915-913.

13. Labeau SO, Van de Vyver K, Brusselaers N, Vogelaers D, Blot SI Prevention of ventilator-associated pneumonia with oral antiseptics: a systematic review and meta-analysis. Lancet Infect Dis 2011;11: $845-854$.

\title{
Detection of influenza myocarditis using national healthcare safety network surveillance definitions accounting for fever in older adults
}

\author{
Rupak Datta MD, $\mathrm{PhD}^{1}$, Elie Helou MD ${ }^{1}$, Mollie Tucker MD ${ }^{1}$, Blessy John MD ${ }^{1}$, Richard A. Martinello MD ${ }^{1,2}$ \\ and Maricar Malinis MD $^{1}$ \\ ${ }^{1}$ Section of Infectious Diseases, Department of Internal Medicine, Yale School of Medicine, New Haven, Connecticut and ${ }^{2}$ Division of Infectious Diseases, \\ Department of Pediatrics, Yale School of Medicine, New Haven, Connecticut
}

To the Editor-Cardiovascular complications following influenza infection are often severe. ${ }^{1,2}$ National Healthcare Safety Network (NHSN) surveillance definitions monitor cardiovascular complications such as myocarditis. However, NHSN surveillance definitions for myocarditis do not account for immunosenescence in older adults. Specifically, older adults frequently have lower baseline temperatures and blunted febrile responses. ${ }^{3}$ Accordingly, Infectious Diseases Society of America (IDSA) guidelines for older adults define fever as (1) a single oral temperature $>100^{\circ}$ $\mathrm{F}$; (2) repeated oral temperatures $>99^{\circ} \mathrm{F}$; or (3) an increased temperature $>2^{\circ} \mathrm{F}$ above baseline. ${ }^{4}$ This is important because older adults incur high risks of influenza-associated complications, ${ }^{5}$ and 2017 NHSN criteria define fever exclusively as temperature $>100.4^{\circ} \mathrm{F}$.

We compared the detection of myocarditis following influenza infection using the 2017 NHSN definition and a proposed modified definition that accounts for fever in older adults. We performed a cohort study of all inpatients $\geq 18$ years of age with influenza A or influenza B between November 1, 2014, and April

Author for correspondence: Rupak Datta, MD, PhD, Section of Infectious Diseases, Yale School of Medicine, PO Box 208022, New Haven, CT 06520. E-mail: rupak.datta@ yale.edu

PREVIOUS PRESENTATION: This work was presented in part at IDWeek 2017 (Abstract no. 64324) in San Diego, California, in the Adult Viral Infection Session (Poster no. 1042) on October 6, 2017.

Cite this article: Datta R, et al. (2018). Detection of influenza myocarditis using national healthcare safety network surveillance definitions accounting for fever in older adults. Infection Control \& Hospital Epidemiology 2018, 39, 1145-1147. doi: 10.1017/ ice.2018.147

(c) 2018 by The Society for Healthcare Epidemiology of America. All rights reserved.
30, 2015, and November 1, 2015, and April 30, 2016, at Yale New Haven Hospital, a 1,541-bed tertiary-care medical center in New Haven, Connecticut. Influenza was detected using real-time Taqman polymerase chain reaction according to the protocol developed by the Yale New Haven Hospital Clinical Virology Laboratory. The Yale Human Investigation Committee approved this study.

We identified all patients developing myocarditis within 30 days following influenza detection using the 2017 NHSN definition and proposed modified definition. The 2017 NHSN definition considered myocarditis as meeting 1 of the following criteria: (1) influenza identified from myocardial tissue or (2) $\geq 2$ clinical features including fever $\left(>100.4^{\circ} \mathrm{F}\right)$, chest pain, paradoxical pulse, or increased heart size with no other recognized cause plus $\geq 1$ additional parameter including an EKG consistent with myocarditis, histological evidence of myocarditis, 4-fold rise in paired sera from IgG antibody titer, or pericardial effusion. Our proposed modified definition had criteria identical to those of the 2017 NHSN definition except for fever, which was defined according to the IDSA guidelines for older adults $\geq 65$ years of age. ${ }^{4}$

For all patients, we recorded demographics, comorbidities, antiviral therapy, receipt of seasonal influenza vaccine, hospital characteristics, all-cause mortality, and whether NHSN criteria were present. Influenza infection was categorized as communityacquired versus hospital-acquired based on detection within 48 hours of admission. We compared differences in the proportions of myocarditis detected with the 2017 NHSN definition versus the proposed modified definition using the McNemar test. 